\title{
Process Knowledge Discovery in Social BPM
}

\author{
Mohammad Ehson Rangiha \\ School of Engineering Computer Science and \\ Mathematics \\ City University London, \\ UK
}

\begin{abstract}
- the utilization of process knowledge for future execution is an effective way of improving the efficiency of business processes and benefit from the knowledge captured in previous executions. This paper attempts to discuss how social tagging can be used in the context of social business process management to assist and support the execution of business processes in a social environment. We believe such an approach is a step forward towards producing a comprehensive model for social business process management.
\end{abstract}

Keywords-Social BPM; BPM; Goal-Based Modelling; Process Recommendation; Social Tagging

\section{INTRODUCTION}

Business Process Management (BPM) is a systematic approach which provides a platform to take full control of the business processes from the beginning until the end. This is achieved through the management, measurement and the improvement of the company processes [2].

With the increase in the usage of social software and user collaboration in the different areas of day to day life, recently traditional BPM models have been influenced by social characteristics which promise to improve and overcome the limitations of traditional BPM approaches. Initial investigations in the area of social BPM has taken place, however these research are still in the initial stages [3]. Social BPM is the intersection between social software and BPM and the integration of social elements into the different stages of the BPM lifecycle.

Goal-based modelling has been proposed as a guiding theory to support social BPM overcome the sequential nature of traditional BPM systems and also enable flexibility during the design and execution of the processes [30]. In this approach goals are defined and set as the intended outcomes to be achieved [6].

The proposed SBPM framework which is inspired by goalbased modelling, does not enforce tasks during the execution of the processes, this has brought about the idea of 'process recommender'. Furthermore process ownership in our SBPM model does not follow a top-down imposing mechanism where the process owners are selected by a higher authority, rather it follows a 'role assignment' approach which is informed by the social behavior of the user and his/her profile static and feedback data [6].

This paper aims to unfold the key elements of the process recommender mechanism in the SBPM model and is structured in the following way:

\author{
Bill Karakostas \\ School of Engineering Computer Science and \\ Mathematics \\ City University London, \\ UK
}

In section 2, the theories used in the formation of the proposed SBPM approach are presented, explaining how they have been adapted in the context of social BPM. This is followed by a high-level model of SBPM illustrating its main components in section 3 in order to position out research at hand accurately.

Section 4 explains the task recommender mechanism, illustrating how it works and presenting a meta-model for this components and introducing its main elements. Furthermore to clarify what has been discussed, an example applied to the theories presented is demonstrated to further clarify the function of the take recommender. Finally, section 5 presents a summary of the research, explains some of the contributions of this approach of SBPM to the field and after identifying some of the limitations of the presented approach, lays the ground for future research and investigation.

\section{Goal-BASEd Social Business Process MANAGEMENT (SBPM)}

\section{A. Overview of BPM}

Business Process Management is a discipline which focuses on optimizing, managing and running of business processes [28] and according to Aalst et al [12] it consists of the following main stages: process design/discovery, configuration/implementation, enactment/execution and evaluation.

Limitations such as Lack of Information Fusion, ModelReality Divide, Information Pass-On Threshold and Lost Innovation and Strict Access-Control [4], have initiated new research such as integrating social characteristics into the BPM lifecycle.

\section{B. Overview of SBPM}

Social Business Process Management is an approach for engaging a range of users in the design, implementation, enactment and evaluation of business processes in order to improve the BPM lifecycle [27]. SBPM is the intersection of BPM with social software to optimize the efficiency of traditional BPM systems [26], and processes are designed and enhanced by adapting various social technologies [8]. Gartner discusses the collaborative nature of social BPM stating that it is a concept which describes collaboratively designed and iterated processes [9]. There has been some research in this area; however none of these have produced a formalized framework yet [3] [5].

SBPM aims to account for the unplanned participation of the users and alteration of the business processes to attain a 
certain level of flexibility and complete enactment of the process steps more efficiently [1]. This is achieved through allowing the users to be part of the design of the processes, therefore instead of a group of analysts designing the processes and passing it onto the end users, the users themselves are also involved in the design of the processes this eliminates the necessity for the presence of a group of process designer [8].

In the current model of SBPM, the analysts and developers are using social software to improve their experience of BPM [8] [26], however social elements have not been fully incorporated throughout the BPM lifecycle and specifically during runtime of the processes. Only recently, [6][30] [26] have proposed goal-based modeling which has been a step forward in formalizing a SBPM framework.

A number of benefits for SBPM has been identified as discussed in [5] such as: exploitation of weak ties and implicit knowledge [1][3], transparency [3][5], decision distribution [3] [16], knowledge sharing [3][16][5]. At the same time, a number of potential limitations and disadvantages have also been mentioned about SBPM, these include: learning effort [4][1], security [4][5], quality [4], difficulty to evaluate [5], process management [26].

\section{Goal-Based Modelling}

Goal- based modelling [31] is the approach which has been used [30] in order to overcome the rigid and sequential nature of traditional BPM systems since flexibility during runtime of the process cannot be achieved with sequential workflows [5]. Goal-based modelling provides a flexible process-flow whilst keeping the integrity of the processes intact in the social BPM framework. Although this approach has been used extensively in requirements engineering [23] [22], however it has had not been adapted in the context of SBPM.

Goals are states which are reached through the execution of a number of steps [28] and they provide the intention behind the activities which need to be performed [30]. The type of goals mentioned here are process goals which are set by the different process areas of an organization and upon completion; their status is changed to 'satisfied'. These goals may well have dependencies and other sub-goals which need to be achieved first before the main goal can be satisfied [29], all of these are modelled in goal-based business process approach.

The goals which are set by the organization are left to the specific user(s) to decide how they would like to achieve them [1] [24]. In other words goal-based modelling allows the capturing of the 'what' without specifying the 'how' as [28] puts it. The 'how' is similar to the imperative approach in which the tasks order and sequence is explicitly defined, and the 'what' is similar to the declarative approach in which the end goal is important and not how it is achieved [68]. These two approaches have been presented in the following figure:

Depending on how detailed the goals are set; the flexibility given to the user to carry out the relevant tasks also varies. Normally what would happen is that after a few iteration of completing a goal, the approach and series of steps which needs to be taken gradually becomes more structured [5]. These goals can then be re-evaluated going forward based on user feedback and experience of running the processes.

In our proposed approach for processes recommendation, the predefined goals are recommended to the user and the user has a degree of flexibility to achieve the proposed goal. Unlike traditional BPM systems, in this approach sequence of actions are not enforced upon the user, rather it only guides the user throughout the enactment of the processes by enabling collaboration and participation from the user community [5].

Giving the user the flexibility to decide how he/she would like to attain a goal would only be feasible in processes where the process flow is not programmed and hard-coded into the system.

\section{OVERALL STRUCTURE OF SBPM}

[7] Presents the architecture of the proposed SBPM framework and outlines the main components of it, namely the task recommender system and role-assignment mechanism. Fig 1presents a high-level view of the various elements in the proposed social BPM model and illustrates how the two main components of our approach is linked with the rest of the environment. Here some of the important parts of this model is explained:

Process Goals: if the overall goal of the process which the users are trying to achieve.

Tasks: each goal have a number of tasks which need to be fulfilled in order for the overall goal to be satisfied. The number or complexity of the tasks are dependent on the nature of the domain specific processes.

Database: static, behavioral and feedback data related to the users and the discussions are all stored in the database and used for recommending tasks and roles as has been presented in [7]

Social User Goals : these are the reasons and motives behind different users involvement and participation in the business processes. 




Fig.1. High-level model of the proposed social BPM framework

The interactions take place in the light of the social environment, which the process recommendation mechanism and role assignment function depend on.

The recommendation engine proposes the various goals which need to be fulfilled to the user and makes these recommendations based on the course of action the user takes.

The role assignment functionality recommends the most suitable user to be responsible for the overall execution of the processes to achieve the specific goals. The method of rating the suitability of the user is explained in later sections.

A typical recommender system has 3 main elements to it, 1. Background data which is the information the system has stored about the user (This is equivalent to the behavioral, profile and feedback data in our proposed model [6], 2. Input data which is the data given to the system as items to be recommended/assigned (Similar to the projects available which need to be recommended to the most suitable user), 3 . Finally an algorithm that combines the background and input data to produce an outcome which is the assignment/proposal of the two data together [21].

\section{TASK RECOMMENDATION IN SBPM}

Recommender systems became popular and an important area of research since the emerging of researches in collaborative filtering in the mid-90s [86]. Recommender systems [20] [19] have been mainly used in e-commerce, content presentation, entertainment and services [19], however they have not been used previously in the context of SBPM.
The recommendation which is discussed here is in the context of social processes, and used as an approach in order to benefit from the process knowledge captured in previous similar business process executions, so it does not use the same algorithms used in Content-based, Collaborative Recommendation and Hybrid approaches [18].

\section{A. Tagging}

Tagging involves the assignment of unrestricted keywords to all kinds of content [11]. These assignments can assist to build up a knowledge repository which contains related useful information on a certain topic. Tagging, for the first time has been proposed to be used in the context of business processes in [10]. This is in order to capture any information used in process execution, and to make these available in the future. This is done through a tagging mechanism of the discussions which take place for the fulfillment of the tasks in a specific process. Social Business Process Management can therefore benefit from such approach to assist and enable improvement to the processes.

Researches [13] in tagging have also categorized tags into different types such as context-based, content-based, subjective and organizational which essentially define what the tags refer to it.

\section{B. Social Tagging Archetecture}

Expanding on previous research [10] carried out in this area, here we define three types of tags which are captured by the system. After the completion of the tasks in any given process, the discussion can be tagged with one or more of the following types: 
<<Process>> : this tag captures the general process in which the discussion is taking place.

$<<$ Task >> : this captures the task which the discussion is about in order to fulfil the overall process goal.

$<<$ Rationale>>: this tag captures the reason and rationale behind tagging a particular segment of the discussion.

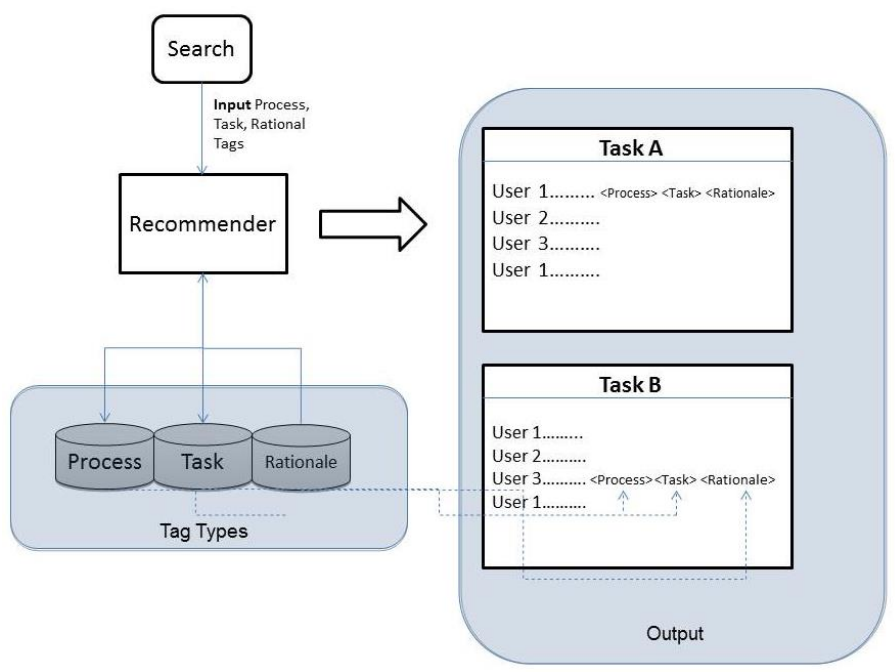

Fig.2. Social Tagging in SBPM

This tagging is done by a process owner [7] who is recommended and chosen based on a number of criteria (interest, expertise and feedback) and is responsible for tagging the discussion with these tags. As demonstrated in fig 2 , there is discussion which takes place by the social user community as part of fulfilling each of the tasks. The process owner who is overall responsible for the completion of the process then tags the discussion which can be useful in future executions. After the completion of the tagging which can be quite challenging depending on the domain, the user who would like to initiate or take part in a similar process in the future is able to utilized the previously captured knowledge by searching a number of key words.

Fig 3 illustrates how the search can be one or more than the type of the tags mentioned above. The user might only be interested in retrieving information which is relevant to the rationale he would like to follow, or he can be more specific and customize the process domain he wishes to look for in which case he will mention the type of the 'process' and 'tasks' which he would like to search.

Based on the input, the system searches through the tags and returns the discussions related to the terms. The discussion and key points mentioned can then be used in the current process which the user is about to run.

With the increase of the number of execution logs and the tags in the system, the process knowledge is expanded and becomes more rich. This will assist the users in the future who would like to partake in similar type of processes.



Fig.3. Process Knowledge Discovery in SBPM

During the discussion, there is a real-time interaction and catering for unplanned participation from the user community which is essential for the SBPM model [9], this interaction model is presented in [6]. The purpose of this is to allow flexibility and a degree of freedom to the user in carrying out the tasks and the set goals.

\section{Proposed Meta-Model for Social Tagging in BPM}

Having explained the mechanism of the task recommender and how it uses social tagging to enable process knowledge discovery, fig 4 presents a meta-model of the proposed approach towards social tagging in the context of social BPM. Essentially, the process owner is a user which is responsible for the tagging of the discussions. These are tagged with different tag types as explained above, and stored in a tag cloud. The user can then use the tag could and enter free text to indicate the type of process of area he is interested in and retrieve the discussion related to the searched terms.

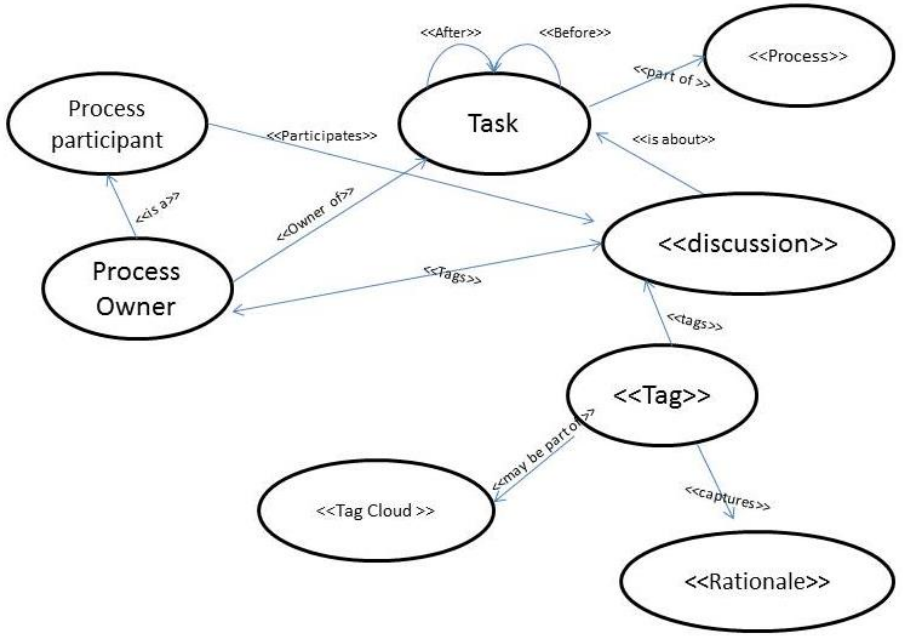

Fig.4. Proposed Meta-Model for Social Tagging

The traditional BPM models do not support the required flexibility and agility for SBPM when it comes to rapid changing and unpredictable process steps; this makes flexibility of the processes during runtime impossible [15]. 
Therefore our proposed recommender process aims to provide a level of flexibility based on the users course of action and guide the user until all the goals are fulfilled. In other words, the users benefit from previously captured process knowledge in order to fulfil the required goal of the process in a semi-structured way without having the process steps imposed on them.

\section{A Worked Example}

To illustrate the theory explained above, consider the process of organizing a football tournament. This process consists of tasks such as booking a pitch, deciding on a time and venue, finding a referee and organizing the match fixtures and so on. After all of these tasks have been collectively accomplished, the process owner revisits the discussions related to each one of these tasks and tags relevant fragments which could be utilized in the future when organizing similar events.

For instance, for the pitch site and venue, after investigating a number of locations and different pitches, the organizing team decided to go with astro turf option. In such an instance, the process owner can tag that segment of the discussion about the choice of the venue with a tag such as 'AstroTurf'. This would be the rationale behind the tagging, he can also use other tags such as 'pitch' to indicate the task which he is tagging and also add the tag 'FootballTournoment' to specify the type of process this tag is for.

Fig 5 demonstrates what has been explained by showing how the tagging will take place after the completion of each of the tasks. The same process will follow for each of the other tasks as well, the rationale maybe different in each case and is left to the process owner to decide. The following is only an example for finding a pitch.

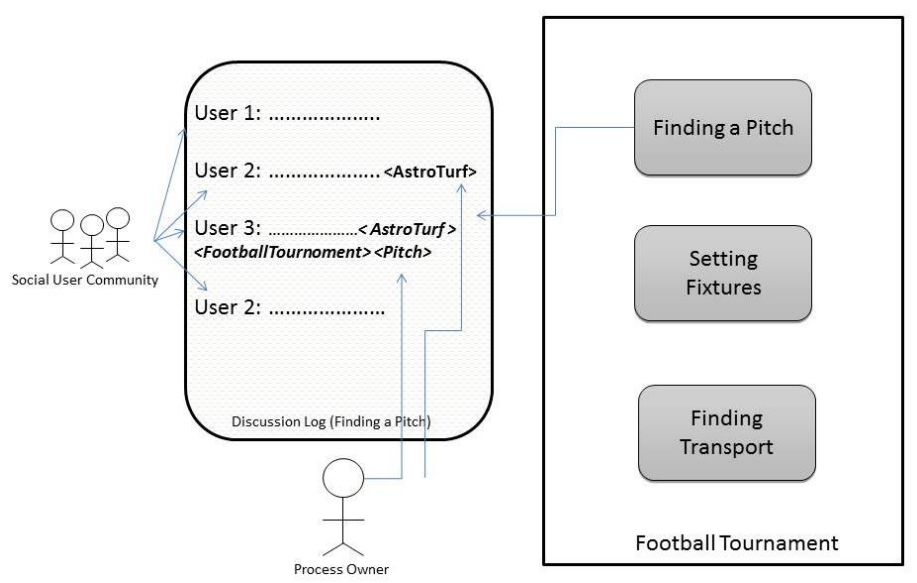

Fig.5. Example of social tagging for a football tournament process

Furthermore, the discussion for the pitch selection can be linked to the discussion and task related to the transportation method to get to the venue. So in the future when someone searches for that specific type of venue, after finding the exact pitch which was decided on, the user can also see the easiest/cheapest/quickest (depending on the tag used for the transportation) to the venue and consider using the same way without the need of having to do the research from scratch.

Thus, not only domain specific knowledge ('AstroTurf) is retrieved but also process specific knowledge (i.e. how to travel to that particular location). The more instances of a process are executed and annotated, the higher the amount of knowledge that will be available for future users to utilize. For their more efficient utilization, previously used tags can be stored in a 'tag Cloud' [17] where the users can see what tags have been previously and their frequencies of use.

\section{CONCLUSION AND REFLECTIONS}

Social BPM research has developed over the past few years and preliminary meta-model [30] has been proposed. This paper unfolded the main components of SBPM which is the task recommendation mechanism to support the user during process execution.

Goal-based approach has been adapted as a way forward for social BPM, the reason for this simply due to the flexibility and control that this approach can bring to the business process management lifecycle.

Goal-oriented SBPM framework is informed by the roleassignment engine and the task recommendation mechanism. The latter was explained in detail throughout this paper, demonstrating how the user is able to benefit and utilize the knowledge captured in past executions. This is done through introducing social tagging to the SBPM model to enable the capturing of process knowledge. These can then be used by users when searching based on a specific criteria they are interested in.

Such an approach truly shifts the BPM model to a socially driven one which is guided by its social context of the users and tasks performed in the past. There is no imposing in such a system and the user and process owners are flexible in the course of actions they wish to take in order to fulfil a specific task or benefit from the captured process knowledge. It was argued that by utilizing the power of social tagging after the completion of the process, future users can benefit from wealth of process related ideas and approaches and reuse them as appropriate. The tagging of the tasks and discussions about them, adds to the whole process knowledge.

The proposed model has also its own limitation which will be addressed in future research. Some of these challenges start from the initial stages of setting the tasks and in the case of conflating goals [25] with tasks (which will reduce the flexibility given to the user), or defining conflicting goals are examples of problems which this approach could face.

The other limitation of the use of social tagging in this context is, the tedious tasks of the process owner tagging the discussion and going through what has been discussed line by line to identify a rationale for some of the segments and tag them. Although the main use of having the <process> and <task> tags are primarily to enable customization during searching, it would be straightforward to do in the text. However identifying the rationale could be challenging and time consuming. 
Different approaches such as 'Fuzzy Logic' [14] could potentially be adapted to be applied in order to assist in the tagging process. Although the accuracy of the tagging might not be the same as manual tagging, however it is an area where further research can be carried out to investigate whether any approach can be used to assist this process.

Furthermore, the problem with free text searching and mismatches has always existed in search engines. This is no different, so although the search mechanism will look through the tagged terms, and find the closest and most relevant match. However, if the incorrect term is used, the user might not be able to get the result her has been looking for. We will try to investigate this further and overcome some aspects of this issue by storing the tags in a tag cloud and make available to the users, so they are able to see all the tagged terms and used them in order to retrieve the process knowledge they are looking for.

Overall, the proposed approach is a step closer to a comprehensive social BPM model which researchers have been talking about for the past few years. Social tagging is used extensively in social media and different social platforms, but it has not been adapted in the context of business process. This model brings about great benefit to the process users and designers and to the overall design, implementation, enactment and evaluation of the business process lifecycle.

The proposed approach towards process knowledge discovery and the role-assignment [7] mechanism need to be validated and applied practically to a real-case study. For this purpose we are in the process of producing a prototype using a wiki in order to implement what has been proposed.

\section{REFERENCES}

[1] Kemsley, S.: Leveraging social BPM For Enterprise Transformation: In Social BPM Work, Planning and Social Collaboration Under the Impact of Social Technology, Swenson, KD., Palmer, N., et al.: BPM and Workflow Handbook Series, pp. 77 - 83, Future Strategies Inc, USA (2011).

[2] R.G. Lee, B.G. Dale, "Business process management: a review and evaluation", Business Process Management Journal, Vol. 4 Iss: 3, pp.214 - 225, MCB UP Ltd (1998).

[3] Brambilla, m., Fraternali, p., Combining social web and BPM for improving enterprise performances: the BPM4People approach to social BPM, In Proceedings of the 21st international conference companion on World Wide Web, pp. 223-226 ACM Publishers, New York (2012).

[4] Filipowska, A., Kaczmarek, M., Koschmider, A., Stein, S., Wecel, K., Abramowicz, w., Social Software and Semantics for Business Process Management - Alternative or Synergy?, in Journal of Systems Integration, vol 2, No 3, pp.54 - 69 (2011).

[5] S. Erol, M. Granitzer, S. Happ, S. Jantunen, B. Jennings, A. Koschmider, S. Nurcan, D. Rossi, R. Schmidt, P. Johannesson. Combining BPM and Social Software: Contradiction or Chance? In Journal of Software Maintenance and Evolution: Research and Practice, vol. 22 pp. 449-476, John Wiley \& Sons, Ltd (2010.)

[6] Rangiha, ME., Karakostas, B.: A Goal-Oriented Social BPM Framework. (Submitted) In: Post-Proceedings of Lecture Notes in Business Information Processing (LNBIP) series, Springer, $15^{\text {th }}$ BPMDS2 workshop in conjunction with CAiSE 14 conference, Thessaloniki, Greece, China (2014).

[7] Rangiha, ME., Karakostas, B.: Process Recommendation and Role Assignment in Social Business Process Management. In: Proceeding The Science and Information Organization, Science and Information
(SAI) Conference, 27 - 29 Aug (2014).

[8] Swenson, K.: Who is Socializing in Social BPM? 12 May 2010 [Blog Entry], Collaborative Planning \& Social Business. Available: http://social-biz.org/2010/05/12/who-is-socializing-in-social-bpm-2/ [Accessed Sep 2014].

[9] Seybod, P.: The next big thing: Adaptive Business Process Management, Users.com, [Blog Entry]: Available at: http://www.users.com/articles/the-next-big-thing-adaptive-businessprocess-management/ [Accessed Oct 2014].

[10] Rangiha, ME., Karakostas, B.: The Use of Social Tagging in Social Business Process Management.(In Print) In: Conference Proceedings The International Conference Business Process Management, London, UK, 12 - 14 Oct (2014)

[11] Cantador I., Konstas I., Jose J.: Categorising Social Tags to Improve Folksonomy-based Recommendations.: In Journal Web Semantics: Science, Services and Agents on the World Wide Web, V.9, Iss. 1, pp. 1 - 15, March (2011).

[12] Rich, E.: User Modeling via Stereotypes. In: Cognitive Science 3, pp. 329-354 (1979).

[13] Panke, S., \& Gaiser, B.: With My Head Up in the Clouds: Using Social Tagging to Organize Knowledge. Journal of Business and Technical Communication, 23(3), 318. (2009)

[14] Lotfi A.Zadeh: Is there a need for fuzzy logic ?.: Science Direct: In information sciences 178, Elsevier Inc, pp. 2751 - 2778 (2008).

[15] Burkhart, T., Loos, P.: Flexible Business Processes - Evaluation of Current Approaches: MKWI '10, Institute for Information Systems (IWi) at the German Research Center for Artificial Intelligence (DFKI. Göttingen (2010).

[16] Richardson, C., Is social BPM a Methodology, A Technology, Or just a lot of Hype? 20 May 2010 [Blog Entry]. Forrester. Available: http://blogs.forrester.com/clay_richardson/10-05-20social_bpm_methodology_technology_or_just_lot_hype[ Accessed Oct 2014].

[17] Surowiecki, J. The Wisdom of Crowds. Abacus; New Ed edition, $1^{\text {st }}$ edition, (2005).

[18] Adomavicius, G.: Toward the Next Generation of Recommender Systems: A Survey of the State-of-the-Art and Possible Extensions. In: IEEE Transactions on knowledge and data engineering, vol 17, no 6 (2005).

[19] Koschmider A., Oberweis A.: Designing Business Processes with a Recommendation-Based Editor, Handbook on Business Process Management 1, pp.299-312 (2010).

[20] Balabanovic, M., Shoham, Y.: Fab: Content-based, collaborative recommendation. In: Communications of the ACM, 40(3):66-72, USA, New York (1997).

[21] Burke, R: Hybrid Recommender Systems: Survey and Experiments: In Journal User Modeling and User-Adapted Interaction, vol 12, issue 4, pp 331-370, Kluwer Academic Publishers Hingham, MA, USA (2002).

[22] Kavakli, E.: Goal-Driven Requirements Engineering: Modelling and Guidance. Available: http://www.ct.aegean.gr/people/vkavakli/publications/pdf_files/PhD/kav akli_chapter2.pdfhype [Accessed Sep 2014]

[23] Yu, E.S.K. and Mylopoulos, J.: Why Goal-Oriented Requirements Engineering. Proceedings of the 4th International Workshop on Requirements Engineering. Foundations of Software Quality (REFSQ '98), pp. 15 - 22. Italy, Pisa (1998).

[24] Dellen, B., Maurer, F., Pews, G.: Knowledge based techniques to increase the flexibility of workflow management. In: Data and Knowledge Engineering, North-Holland (1997).

[25] Adams, M., Hofstede, A., Edmond, D., van der Aalst, W.: Facilitating Flexibility and Dynamic Exception Handling in Workflows Through Worklets. In: Proceedings of the CAiSE '05 Forum, pp. 45-50, FEUP (2005).

[26] Rangiha, ME., Karakostas, B.:Goal-Driven Social Business Process Management. In: Proceeding The Science and Information Organization, Science and Information (SAI) Conference, pp. 894 - 901, London, UK (2013). 
[27] Nurcan, S., Etien, A., Kaab, R., \& Zouka, I.: A strategy driven business process modelling approach. Journal of Business Process Management, 11, 6, pp. 628-649 (2005).

[28] Filipowska, A., Kaczmarek, M, Kowalkiewicz, M., Zhou, X., Born, M.: Procedure and guidelines for evaluation of BPM methodologies: In Business Process Management Journal, vol. 15 Iss: 3, pp.336 - 357 (2009).

[29] Dellen, B., Maurer, F., Pews, G.: Knowledge based techniques to increase the flexibility of workflow management. In: Data and
Knowledge Engineering, vol 23, Issue 3, pp. 269-295, North-Holland (1997).

[30] Rangiha, ME., Karakostas, B.: Towards a Meta-Model for Goal-Based Social BPM. (In press) In: Post-Proceedings of Lecture Notes in Business Information Processing (LNBIP) series, Springer, 6th BPMS2 workshop in conjunction with BPM 2013 conference, Beijing, China (2013).

[31] Kueng, p., Kawalek, P.: Goal-Based Business Process Models: Creation and Evaluation: In Business Process Management Journal Emerald, vol.3, no.1, pp. 17-38. MCB University Press (1997). 